\title{
Bone of the Extremity
}

National Cancer Institute

\section{Source}

National Cancer Institute. Bone of the Extremity. NCI Thesaurus. Code C32223.

A bone within a limb of the body. 